Running Head: COSTS AND BENEFITS OF METAPHOR

The Costs and Benefits of Metaphor

\author{
Ira A. Noveck \\ Centre Nationale de Recherche Scientifique, \\ Institut des Sciences Cognitives, Lyon \\ \& Université de Grenoble
}

Maryse Bianco

Université de Grenoble

Alain Castry

Université de Grenoble

From Metaphor and Symbol, 16(1\&2), 109-121. 
Many researchers consider metaphor so fundamental to psychological activity that they claim that it does not require extra cognitive effort to process. While not disputing that metaphors are natural to human cognition, we argue that a metaphor's relative ease-of-use ought not be confounded with an expectation that it prompts no extra effort. As many studies show (including those presented here), metaphors often come with costs when compared to non-figurative controls (e.g. longer processing times). However, we also argue that the extra costs associated with an apt metaphor ought to come with benefits. This analysis, based on Relevance Theory, does a good job of accounting for some overlooked psycholinguistic findings concerning metaphor processing. 


\section{The Costs and Benefits of Metaphor}

In this paper, we address two accounts of metaphor comprehension. One comes from Gibbs (1994) who, in keeping with Lakoff and Johnson's (1980) seminal view, argues that metaphor is not a special rhetorical device but fundamental to human cognition. He theorizes that metaphors serve to map one conceptual domain to another in a reflex-like manner that does not require special cognitive effort. The other approach, based on Relevance Theory (Sperber and Wilson, 1986/1995), actually shares much with Gibbs's account. Relevance Theory too does not consider metaphor to be a special device while viewing metaphor as natural to human cognition. Furthermore, like Gibbs's account, Relevance Theory resists a classical Gricean or Searlean analysis which assumes that a listener first needs to reject a literal interpretation of a metaphor in order to appreciate the metaphor's meaning. Relevance Theory differs in that cognitive effort is central to its analysis of utterances in general. Based on a Relevance theory analysis, we argue that it is reasonable to expect metaphors to require more effort relative to a non-figurative equivalent.

In what follows, we briefly describe Relevance Theory and a cost/benefit explanation of metaphors that the theory inspires. We then review data drawn from a paradigm developed by Gibbs which indicate that costs are indeed evident in metaphor processing. Albeit to a lesser degree, his data also reveal some benefits for metaphoric processing. Before concluding, we present two developmental studies that buttress our claims based on Gibbs's paradigm. Our ultimate aim is to show how Relevance Theory can do a good job of accounting for psycholinguistic findings on metaphor.

\section{METAPHORS AS EFFORT-IMPOSING LOOSE-TALK}

Relevance Theory views inference-making as a constant feature of communication geared towards gathering in (and sharing) one's intentions. Essential to Relevance Theory is the claim that, in processing any utterance, people endeavor to draw out as many cognitive effects (i.e. benefits) as possible for the least effort (i.e. cost). Two features of Relevance Theory are crucial for describing metaphors (see Sperber and Wilson, 1986/1991): A) Utterances need not be literally true in order for a listener to draw implications effectively (which is why metaphors are considered a form of loose-talk) and; B) A metaphoric utterance is likely to carry more information than a more-literal, more-direct equivalent. To illustrate, consider a scenario in which a swimming instructor says to a 5-year-old, you are a tadpole. The utterance is a) not literally true while effectively conveying information from teacher to student and it b) goes further than its literal equivalent (which 
one can presume is something like you are a young child doing a frog kick); at the very least, the instructor's expression is arguably endearing whereas the literal equivalent is not.

It is this second feature that concerns us here because it is related to the listener's comprehension of utterances. Relevance Theory essentially argues that the metaphor prompts multi-tasking. In the you are a tadpole example, the metaphor is making reference to the swimming student plus it is describing something about him and transmitting affection; a neutral expression like you are a child in the same context would be doing the reference portion only (and would not seem terribly informative). This comparative analysis leads us to hypothesize that there are (at minimum) two components to a full appreciation of metaphor: Understanding what the metaphor is referring to and understanding the interlocutor's intention in using it. Being that it is difficult to imagine that the extra cognitive effects associated with metaphor come cost-free, we are led to make two claims. Our primary claim is that it should not be surprising to find evidence supporting the notion that -all other things being equal -- metaphors are costly when compared to literal controls. Our auxiliary claim is that the extra costs that come with an apt metaphor ought to be commensurate with extra benefits. That is, one should be able to find evidence indicating that appreciated metaphors have benefits when compared to nonfigurative equivalents.

The above analysis brings together three strands of metaphor research that might, at first glance, appear unrelated. One concerns studies showing that compared to literal controls, there are costs associated with metaphors either in terms of comprehension among children (Vosniadou, Ortony, Reynolds, \& Wilson, 1984) or in terms of relatively longer reading times for metaphoric over synonymous formulations among adults (e. g. Healy and Gerrig, 1983). A second comes from Williams-Whitney, Mio, and Whitney (1992) who establish a link between available effort and metaphor-production. They show that third- and fourth-year college students are more likely than a select group of first-year students to produce novel metaphors in writing about an imaginary character or about themselves. That is, as more resources become available with age or perhaps skill, novel metaphors are more likely to crop up. The third strand is based on work indicating that metaphors have implicit benefits that can lead to deeper encoding of materials. Reynolds and Schwartz (1983) show how a paragraph having a metaphoric conclusion, as opposed to a literal one, consistently leads to higher (immediate and delayed) "memorability" of both the conclusion and its context. These three strands of research taken together indicate that metaphors are costly but they have the potential to provide benefits. Our experiments will attempt to demonstrate this while employing one overarching procedure. 


\section{GIBBS'S METAPHORIC REFERENCE PARADIGM}

We turn to Gibbs's account on metaphor processing and begin by noting his skepticism about Relevance Theory's approach. In his book, Gibbs writes:

[The] psychological research ... clearly shows that listeners do not ordinarily devote extra processing resources to understanding metaphors compared with more literal utterances. The metaphor-as-loose-talk view ... incorrectly assumes that metaphors, and other tropes such as irony, obligatorily demand additional cognitive effort to be understood (Gibbs, 1994, p. 232).

Part of Gibbs's claim about the ordinary nature of metaphor processing comes from his own paradigms. Consider the findings from the following experiment in which metaphors are shown to be as efficient as nonmetaphors in making reference to a previously-mentioned item in a text (Gibbs, 1990). Gibbs presented participants with eight lines of a story before presenting one of three different concluding sentences that vary with respect to their referential content. For example, the story (1a-h) below concerns a weak boxer and a boxing match and the lines (2-4) were one of its concluding sentences:

(1) (a) Stu went to see the Saturday night fights.

(b) There was one boxer that Stu hated.

(c) This guy always lost.

(d) Just as the match was supposed to start, Stu went to get some snacks.

(e) He stood in line ten minutes.

(f) When he returned, the bout had been canceled.

(g) "What happened?" Stu asked a friend.

(h) The friend replied,

(2) The creampuff didn't even show up.

(3) The fighter didn't even show up.

(4) The referee didn't even show up. 
Sentences (2) and (3) are, respectively, metaphoric and synonymous references to the same character, the hated boxer. Sentence (4), which makes reference to a previously unmentioned referee, served as a control. Gibbs found that the average reading time for control sentences like (4) was not significantly different from the reading times of (2) or of (3). Thus, Gibbs was not convinced that metaphors required relatively more effort to process. He also gave prominence to a probe task, in which the participant is required to determine quickly whether a particular word appeared previously in the story. A relatively quick "yes" response to the earlier-instantiated referent of the metaphoric term could be a measure of processing benefit. For example, a "yes" response to the word "boxer" after reading (2) above would signal that the metaphor served to prime the referent. Although Gibbs found a facilitation for probes following metaphoric conclusions $(1118 \mathrm{msec})$ compared to the control condition's conclusions $(1331 \mathrm{msec})$, he also found that the synonymous condition's probes led to latencies (1229 msec) that were statistically comparable to the metaphoric condition's probes. Thus, he took the metaphoric references to be as efficient as synonymous ones.

The results from this experiment actually underline how the comprehension of metaphors appears to require some effort and appear to provide some benefits. We point to two of his main findings. First, the mean reading time of Gibbs's control sentences is actually intermediate $(1867 \mathrm{msec})$ between metaphoric sentences like (2) above (2117 msec) and synonymous sentences like (3) above (1735 msec); the analysis as reported did not capture the distinction of interest, which is that a sentence containing a metaphoric reference took significantly longer to read than its synonymous counterpart. Comparisons to the control sentence, which in the case of (4) leads to different kinds of implications than those conveyed by referring to the boxer, are far less revealing than the metaphor/synonym distinction. Second, reactions to the probes in the metaphor condition tended to be faster (and less error-prone) than those in the synonymous formulation condition. Gibbs's findings suggest that there are processing costs associated with metaphoric references, but that these terms have the potential to provide benefits as well.

\section{THE EXPERIMENTS}

To investigate our account of metaphor comprehension, we have adopted Gibbs's reference paradigm described above. We began with two goals. One was to verify that unanticipated metaphoric formulations do indeed require extra effort when compared to synonymous ones as measured through reading times (the finding that Gibbs tended to disregard). ${ }^{1}$ The other was to determine whether we can find evidence that a by-product of 
extra effort is added benefits. In order to test our hypotheses, we investigated development. Aside from the fact that minimal competence with metaphors among children would add support to claims favoring the naturalness and ubiquity of metaphor, we anticipated that evidence of effort ought to be even clearer among younger readers.

We now make two concrete predictions based on the supposition that there are at least two components to a full appreciation of metaphor: (a) detecting the referent of the metaphor and (b) comprehending an added effect, e.g. something that tells the reader more about the author's or story-character's intention. When faced with an informationally rich metaphoric reference instead of a synonymous one, we argue that all readers are compelled to do extra processing. Thus, there should be evidence showing that all readers require more time to understand a metaphoric reference than a synonymous control. For our second prediction, assume that both a child and an adult have a fixed amount of time (say a half-second) to integrate a read line of text with previous information. In that fixed amount of time, an adult should be able to integrate more information (references, intentions, attitudes, etc.) than a younger one. Compared to a synonymous reference, the appearance of the metaphor (the imposed extra effort) risks undermining an immature reader's comprehension with respect to (a) or to (b) or to both. In contrast, the appearance of the metaphor for a competent reader ought to lead to the attainment of both (a) and (b), leading to a richer appreciation for the metaphoric reference, compared to the synonymous equivalent. This analysis ought to be reflected by rates of correct responses to follow-up questions. Because of space limitations we summarize briefly the findings from two experiments .

\section{General Method}

The stories in our experiments were modeled on Gibbs's paradigm described above with one modification: we avoided placing the metaphoric or synonymous references in the concluding line. Sixteen eight-line texts were prepared that had, as the penultimate line, a sentence containing either a metaphoric or synonymous reference to a previously mentioned item. For example, consider the (translated) story in (5) below and the metaphor in the second to last line (line $5 \mathrm{~g}$ ):

(5) (a) The second-grade pupils went to the pool with their teacher.

(b) The lifeguard organized a few games for them.

(c) He then asked that they do a few laps.

(d) Before the end of the class, the phone rang. 
(e) The lifeguard went to answer it.

(f) Returning, he cried out:

(g) "All toads to the side of the pool."

(h) The class went to the lockers and back to school.

In the synonymous formulation condition, the second to last line $\left(5 g^{\prime}\right)$ read as:

(g') "All students to the side of the pool."

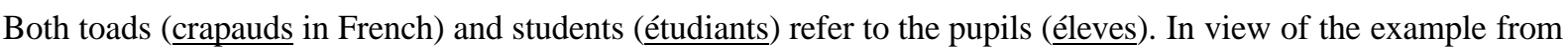
Gibbs's paper, we included conventionalized metaphors among our stories but we tried to avoid them (crapauds is an example of a conventionalized metaphor in French).

Experiment 1. Two hundred and thirty children between the ages of 8 and 12 were presented the 16 stories on paper. Table 1 includes a list of the sixteen referred terms, metaphors and synonyms used. The stories were divided up into in two sets, A and B. Those who received the metaphoric versions of the stories in set $\mathrm{A}$ received the synonymous versions of the stories in set B, and vice-versa. Four random orders were prepared. All the stories provided questions that directly asked about the referent and all the questions required a "yes" or "no" response. For example, with respect to (5) above the question (presented while the text was available) was Were the pupils the ones who went to the side of the pool? Regardless of formulation (metaphoric or synonymous), the correct response is "yes".

Insert Tables $1 \& 2$ about here

Table 2 presents a summary of the results from the first experiment. A 5 (Age: 8, 9, 10, 11, 12) X 2 (Reference Type: synonymous vs. metaphoric reference) ANOVA with repeated measures on the second factor was conducted. We found two revealing effects and no interaction. First, there was a main effect for age, $F(4,75)=2.771, p<.05$. This shows that referential ability in general is improving with age. Second, formulations containing synonymous references consistently prompted higher rates of correct responses than those containing metaphoric ones, $F(1,75)=22.852, p<.0005$. In fact, formulations with synonymous references provide rates of 
correct responses that are consistently about 7\% higher than those with metaphoric ones until around 12 years of age. Among 12-year-olds, one sees the gap between metaphoric vs. synonymous reference comprehension closing -- the 12-year-olds showed that formulations with synonymous references led to rates of correct performance that were only $2.6 \%$ superior to those prompted by formulations having metaphoric references (94.9\% vs. $92.3 \%)$. This indicates that metaphors do come with a small risk of leading young readers astray but the risk appears to diminish progressively with age.

All responses are clearly above chance levels so one can conclude that children are quite competent at detecting and accounting for a metaphoric reference; however, these results indicate that unexpected metaphors do impose a burden on the reader. This would account for the slight, consistent advantage (in terms of rates of correct responses) associated with stories containing the synonymous formulations among younger readers. Note that this effect is evident despite the fact that children have full access to their texts.

Experiment 2. In order to further substantiate our claim that metaphoric references indeed impose a burden and thus take additional effort to process, we introduced an on-line version of this task. There were 50 nine-year-olds, 48 eleven-year-olds, 51 fourteen-year-olds and 40 adults. The nine-, eleven- and fourteen-yearolds were presented 12 of the sixteen stories from Experiment 1. Adults were presented all sixteen stories from Experiment 1 plus filler items (concerning deductive inference). Thus, the adult data came from a more demanding session. All analyses will concern only those stories that were seen by both the children and adults.

As in Experiment 1, the stories were divided up into in two sets, A and B. Those who received the metaphoric versions of the stories in set A received the synonymous versions of the stories in set $\mathrm{B}$, and viceversa. The number of participants who received set A was roughly equal in size to the number who received set B for all age groups. As in Gibbs's original paradigm, the stories were presented randomly one line at a time by computer in a self-paced manner and the participants' reading times for the metaphoric and synonymous formulations were measured. The materials were reworked only slightly to verify that sentence length of the critical line (like 5g's) was roughly equivalent across stories (between 9 and 13 syllables). Each story was accompanied by one of three kinds of follow-up questions requiring a "yes" or "no" response: a) a question about a detail of the story, b) a general comprehension question, or c) a question like the one in the first experiment concerning the referent. Only one kind of question was presented after each story, but for example, these three kinds of questions about the story in (5) above would be: Were the students in the second-grade? (detail), Was the lifeguard interrupted during the class? (general comprehension), Were the pupils the ones to go to the side of 
the pool? (referent). Unlike in this example, correct responses often required "no" responses. Note that this reading task is more difficult than the one in the first experiment (especially for younger readers) in that memory load is more critical here.

We analyze the results concerning the reading-time of the crucial penultimate line as well as correct responses to the questions posed. We point out that one story was removed from all analyses because a programming error presented the story (the one using referent 9 in Table 1) in its metaphoric guise throughout to the children. We also note that we did not filter out reading times, i.e. all reading times from the 11 remaining stories were included in the analysis. Two other stories (stories for referents 14 and 15) were removed from the analyses concerning rates of correct responses only because the questions were not clear and led to responses that were difficult to interpret (e.g. one question was "Grenouillette doesn't have much imagination?" when the story indicated that she did; either one of the response options -- "yes" or "no" -- does not capture a correct response). A summary of the results is presented in Table 3.

\section{Insert Table 3 about here}

We turn first to the Reading Time results. A 4 (Age: 9, 11, 14, Adults) X 2 (Reference Type: synonymous vs. metaphoric reference) ANOVA with repeated measures on the second factor was conducted. The results revealed a main effect for Age, $F(3,40)=53.6, p=.0001$, a main effect for Reference Type, $F(1$, 40) $=44.8, p=.0001$, and an Age X Reference Type interaction, $F(3,40)=7.6, p<.0005$. It is not surprising that reading speed increases with age. More interesting is that, at each age, one finds that sentences containing the metaphoric reference are read consistently more slowly than those containing the synonymous control and that the gap closes with age, though never completely. The adult data confirm that metaphoric reference in Gibbs's paradigm prompts a slow-down when compared to synonymous controls.

To analyze rates of correct responses a 4 (Age: 9, 11, 14, Adults) X 2 (Reference Type: synonymous vs. metaphoric reference) ANOVA with repeated measures on the second factor was conducted. The only effect that even approached significance was the Age X Reference Type interaction, $F(3,32)=2.76, p=.058$. The interaction is an indication of evolving benefits with age. The rates of correct responses show that the youngest children, like in the first experiment, pay a small price in comprehension when they encounter a metaphoric reference. Adults reveal that the metaphoric reference actually aids comprehension slightly. We remain 
cautious about our second finding because, as indicated above, the adult data were collected from an experiment that also included filler stories. Nevertheless, the pattern of results is consistent with the cost/benefit analysis that led to our initial predictions.

To summarize, Experiment 1 showed that for the growing reader it is easier to make links with a previously-mentioned term (to the referent) when the reference is synonymous with the term rather than metaphoric. Experiment 1 also indicated that this advantage decreases with age. Experiment 2 revealed the extent of this developmental effect. It showed that for the youngest children the rate of correct responses to follow-up questions was lower when a metaphoric reference had been used, as in Experiment 1; for the adults the rate of correct responses to follow-up questions was higher when a metaphoric reference had been used. The youngest children seem to suffer somewhat when faced with the metaphor and adults seem to benefit. Experiment 2 also showed that, compared to synonymous controls, metaphoric references are consistently associated with relatively longer reading times.

\section{CONCLUSIONS}

While drawing on Relevance Theory, we anticipated that the comprehension of a metaphoric reference is more demanding than that of a synonymous one, i.e. that there is an extra cost in processing a well-chosen metaphor. Universally longer reading times for sentences containing unanticipated metaphoric references is one piece of evidence revealing of costs. A second is younger readers' lower rates of correct responses when questions followed a metaphoric reference instead of a synonymous one. However, the metaphoric reference has the potential to yield benefits. The slightly higher rates of correct responses among adults when questions followed a metaphoric reference instead of a synonymous one is an indication that metaphors offer multiple effects.

We consider our data pointing to costs clear. Evidence in favor of benefits is less abundant. This is partly due to the fact that our follow-up questions did not necessarily tap into unique aspects of each metaphoric reference. For instance, an added effect of the toads reference in $(5 \mathrm{~g})$ might well be something related to affection whereas the added effect of another metaphor (consider tank as a reference for vacuum-cleaner) might be descriptive (that it is loud and clunky). In both cases, the story's follow-up question did not touch upon the invited features of the respective metaphors. We predict clearer support for our claims about benefits when the metaphor's implicit effects can be specified for each individual case. If one does not find stronger supporting 
evidence for benefits when the follow-up questions are better controlled, it would present a challenge for our account. Generally speaking, to undermine our analysis one would have to show that an unanticipated, apt, and appreciated metaphor violates our cost/benefit analysis. This demonstrates how our application of Relevance Theory is testable.

How would Relevance Theory account for those cases that indicate less effort (e.g. faster reading times relative to non-figurative controls) for sentences or words that had been used metaphorically? We respond by taking a careful look at a set of studies by Allbritton, McKoon and Gerrig (1995) who showed that a sentence like Both sides were now bringing out their heavy artillery was read significantly faster (as a target in a priming task after it had been read once in a story) when it had been a metaphoric conclusion rather than a plausible literal conclusion. We make two points. First, when a metaphoric formulation is shown to be advantageous compared to a literal one (in terms of faster reading times), it is arguably due to important changes in their respective contexts. For example, in Allbritton et al.'s Experiment 1, the condition that rendered metaphoric Both

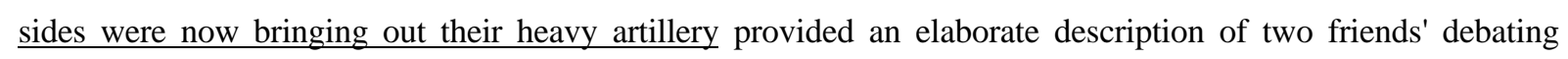
tendencies making this sentence readily understood. As the authors noted, the control condition was potentially less clear; comprehension of this critical line could have suffered from an earlier change in topic. The upshot is that the context for the primed sentence was sufficiently rich for accessing the metaphor in the metaphoric condition and arguably obscure in the control condition. ${ }^{2}$ Second, their priming study allows one to tap into encoding. Thus, much like in Reynolds and Schwartz (1983), the Allbritton et al. work can be taken to show that the clearly signaled metaphor provided some benefits to the participant when it was first read and that these carried over to the priming study. Gibbs's (1990) probe results can be similarly construed.

That metaphoric comprehension can be easily affected by context is well known. Cues need only be minimal in order to reduce the effort involved in comprehending metaphors (see Pynte, Besson, Robichon \& Poli, 1996, who show this nicely with the aid of evoked-potentials). Any claims about effort are relative to a provided context. The present work shows that metaphors can be seen to be costly in contexts that are arguably neutral otherwise.

To sum up, we have pointed out that Gibbs, among others, gives the strong impression that metaphors are not costly. We were skeptical of this claim, especially if the implication is that literal expressions are serviceable replacements for their metaphoric counterparts. We have investigated metaphoric references in order to detail our argument. We have shown that results from Gibbs's study support our contention that metaphors 
come with some processing cost. Inspired by an alternative approach, Relevance Theory, we have argued that metaphors can be analyzed in terms of costs and benefits. Our findings show that a) compared to controls, metaphoric references consistently prompt longer reading times and b) in terms of comprehension, metaphoric references are sources of difficulty for younger children and sources of potential benefit for adults. We thus hope to have shown that a metaphoric reference is an imposition on a reader but that its potential for impact is linked with an ability to appreciate its intended meaning. 
Notes

1 If too many cues anticipate the metaphor, the metaphor would be effectively primed and its informational impact would be reduced. For example, the metaphoric appeal of (and difficulty in comprehending) "You are a piglet" would be diminished if the referent was anticipated in a context stuffed with farms, pens, mud, youngsters, oinking, etc. Our paradigm tried to avoid such scenarios.

2 To address this issue of obscurity, their final experiment provided only the richly described metaphoric condition and presented either the metaphoric conclusion or a neutral non-figurative conclusion. They found that a key term in their metaphoric condition served as a better prime (e.g. artillery) for a target word (e.g. battling) than did a key term from a neutral control (e.g. points). However, the metaphoric term was arguably endowed with richer meaning than the neutral term because the entire paragraph referred to the notions critical to the metaphor (debates and combat etc.). Thus, the comprehension of the metaphoric term came with more benefits than the neutral one in the first phase of the experiment. This was arguably demonstrated when the metaphor was later used as a prime. 


\section{References}

Allbritton, McKoon, \& Gerrig (1995). Metaphor-based schemas and text representations: Making connections through conceptual metaphors. Journal of Experimental Psychology: Learning, Memory and Cognition, 21, No. 3, 612-625.

Gerrig, R. J. \& Healy, A.F. (1983). Dual processes in metaphor understanding: Comprehension and appreciation. Journal of Experimental Psychology: Learning, Memory and Cognition, 9, No. 4, 667-675.

Gibbs, R. (1990). Comprehending figurative referential descriptions. Journal of Experimental Psychology: Learning, Memory and Cognition, 16, No. 1, 56-66.

Gibbs, R. (1994). The Poetics of Mind: Figurative Thought, Language, and Understanding. New York: Cambridge University Press.

Lakoff, G. and Johnson, M. (1980) Metaphors we live by. Chicago: University of Chicago Press.

Pynte, J. Besson, M. Robichon, F.-H., \& Poli, J. (1996). The time-course of metaphor comprehension: An eventrelated potential study. Brain and Language, 55, 293-316.

Reynolds, R. E. \& Schwartz, R. M. (1983). Relation of metaphoric processing to comprehension and memory. Journal of Educational Psychology, 75, No. 3, 450-459.

Sperber, D. \& Wilson, D. (1986). Loose Talk. Proceedings of the Aristotlean Society, 86 (1985-1986), $153-171$. [Reprinted (1991) in S. Davis (ed.) Pragmatics: A reader, 540-549. NY: Oxford University Press].

Sperber, D. and Wilson, D. (1986/1995). Relevance: Communication and Cognition. Blackwell, Oxford.

Vosniadou, S., Ortony, A., Reynolds, R.E. \& Wilson, P.T. (1984). Sources of difficulty in children's comprehension of metaphorical language. Child Development, 55, 1588-1607.

Williams-Whitney, D., Mio, J. S., \& Whitney, P. (1992). Metaphor production in creative writing. Journal of Psycholinguistic Research, 21, No. 6, 497-509. 


\section{Authors' note}

We very much appreciated the comments and the discussion that followed from the presentation of this work at the AISB conference in Edinburgh. We would like to thank John Barnden, Ingar Brinck, Dick Carter, Craig Hamilton, David Nicolas, Dan Sperber, and two anonymous reviewers for some very helpful comments on an earlier draft. Address all correspondence to Ira Noveck, Institut des Sciences Cognitives, 67 Boulevard Pinel, 69675 Bron, France (email: noveck@isc.cnrs.fr). 


\section{Table 1}

List of initial referents and their respective metaphoric and synonymous references as used in Experiment 1.

\begin{tabular}{|c|c|c|}
\hline Initial Referent & Metaphoric & Synonymous \\
\hline & reference & reference \\
\hline 1. animateur & perroquet & présentateur \\
\hline 2. élèves & crapauds & enfants \\
\hline 3. aspirateur & $\operatorname{tank}$ & machine \\
\hline 4. écharpe & serpent & cache-nez \\
\hline 5. Jean-Pierre & gorille & maître-nageur \\
\hline 6. chat & fauve & félin \\
\hline 7. héron & requin & oiseau \\
\hline 8. projecteur & bécane & appareil \\
\hline 9. automobile & baignoire & voiture \\
\hline 10. Marie et ses copains & étourneaux & petits \\
\hline 11. la mère du président & pie & dame \\
\hline 12. avion & rapace & appareil \\
\hline 13. autruche & tornade & oiseau \\
\hline 14. éléphante & bombe & bête \\
\hline 15. citrouille & monstre & potiron \\
\hline 16. flute & rossignol & instrument \\
\hline
\end{tabular}

Notes. References for 2, 8, and 11 are conventional metaphors and those in 5 and 15 can be construed as such in appropriate contexts. 


\section{Table 2}

Percentage of correct responses to reference question among children who are between 8 and 12 years of age.

\begin{tabular}{|c|c|c|c|c|c|}
\hline \multirow{3}{*}{$\underline{\text { Reference }}$} & \multicolumn{5}{|c|}{ Age } \\
\hline & 8 & 9 & 10 & 11 & 12 \\
\hline & $(n=41)$ & $(\mathrm{n}=51)$ & $(n=46)$ & $(n=41)$ & $(n=51)$ \\
\hline Metaphoric & 79 & 85 & 88 & 87 & 92 \\
\hline Synonymous & 87 & 90 & 95 & 94 & 95 \\
\hline
\end{tabular}

Notes. An example of a reference question is Were the pupils (élèves) the ones who went to the side of the pool? where the metaphoric reference was toads (crapauds) and the synonymous reference was students (étudiants). 


\section{Table 3}

Children's and adults' mean speed of reading the line containing either a metaphoric or synonymous reference (plus the mean rates of correct responses to stories' subsequent question).

\begin{tabular}{lccc}
\hline \multicolumn{1}{c}{ Age } & $\underline{\mathrm{n}}$ & $\underline{\text { Metaphoric }}$ & \\
& & $\underline{\text { Reference Type }}$ & \\
& & & \\
9-year-olds & 50 & $4908 \mathrm{msec}(74 \%)$ & $5586 \mathrm{msec}(82 \%)$ \\
11 -year-olds & 48 & $3609 \mathrm{msec}(86 \%)$ & $3842 \mathrm{msec}(77 \%)$ \\
14 -year-olds & 51 & $2851 \mathrm{msec}(90 \%)$ & $2967 \mathrm{msec}(87 \%)$ \\
Adults & 40 & & $2321 \mathrm{msec}(83 \%)$ \\
\hline
\end{tabular}

Notes: Reading times are based on 11 texts and rates of correct responses are based on 9 of these. Unlike the children, the adults viewed filler items. See text for details. 